\title{
Regulate and be regulated: integration of defense and other signals by the AtMYB30 transcription factor
}

\author{
Sylvain Raffaele ${ }^{1,2}$ and Susana Rivas ${ }^{1,2 *}$ \\ 1 INRA, Laboratoire des Interactions Plantes-Microorganismes, UMR441, Castanet-Tolosan, France \\ ${ }^{2}$ CNRS, Laboratoire des Interactions Plantes-Microorganismes, UMR2594, Castanet-Tolosan, France
}

\author{
Edited by: \\ Laurent Deslandes, Centre National \\ de la Recherche Scientifique, France \\ Reviewed by: \\ Volkan Cevik, University of Warwick, \\ UK \\ Roberto Solano, Centro Nacional de \\ Biotecnologia-Consejo Superior de \\ Investigaciones Cientificas, Spain \\ *Correspondence: \\ Susana Rivas, Laboratoire des \\ Interactions \\ Plantes-Microorganismes, UMR \\ CNRS/INRA 2594/441, 24 Chemin \\ de Borde Rouge-Auzeville, CS \\ 52627, Castanet-Tolosan cedex \\ 31326, France. \\ e-mail: susana.rivas@toulouse.inra.fr
}

Transcriptional regulation in host cells plays a crucial role in the establishment of plant defense and associated cell death in response to pathogen attack. Here, we review our current knowledge of the transcriptional control of plant defenses with a focus on the MYB family of transcription factors (TFs). Within this family, the Arabidopsis MYB protein AtMYB30 is a key regulator of plant defenses and one of the best characterized MYB regulators directing defense-related transcriptional responses. The crucial role played by AtMYB30 in the regulation of plant disease resistance is underlined by the finding that AtMYB30 is targeted by the Xanthomonas type III effector XopD resulting in suppression of AtMYB30-mediated plant defenses. Moreover, the function of AtMYB30 is also tightly controlled by plant cells through protein-protein interactions and post-translational modifications (PTMs). AtMYB30 studies highlight the importance of cellular dynamics for defense-associated gene regulation in plants. Finally, we discuss how AtMYB30 and other MYB TFs mediate the interplay between disease resistance and other stress responses.

Keywords: Arabidopsis, AtMYB30, hypersensitive response, MYB transcription factor, plant defense, stress responses, transcriptional regulation

\section{INTRODUCTION}

As sessile organisms, plants must face the diversity of pathogens that they encounter in their habitat. Unlike mammals, plants rely on cell autonomous innate immunity and on systemic signals originating from infection sites (Jones and Dangl, 2006). Plant immunity is activated by multiple transcriptional regulators that switch cell transcription programs from routine cellular requirements to defense. The arsenal of transcriptional regulators includes DNA-binding transcription factors (TFs) and proteins that regulate these TFs. Plant transcriptional regulators function cooperatively in complex networks to control the speed, intensity, localization, and duration of the immune response (Moore et al., 2011). The rapid and localized programmed death of infected cells is part of a typical plant immune response designated as the Hypersensitive Response (HR) (Mur et al., 2008; Coll et al., 2011). Processes related to the sessile lifestyle of plants have been associated with the expansion of TF families controlling plantspecific functions (Dias et al., 2003; Shiu et al., 2005; Feller et al., 2011). The MYB family of TFs underwent an extensive amplification approximately 500 million years ago due to recent whole-genome duplications and segmental tandem duplication events (Shiu et al., 2005). As a result, the plant MYB family typically comprises hundreds of members, classified based on the number of MYB repeats that they contain (Feller et al., 2011). MYB R2R3 proteins contain two MYB repeats and form the largest group of MYB TFs in plants. Members of the R2R3 MYB family regulate mostly plant-specific functions, including immunity against microbial pathogens (Stracke et al., 2001; Dubos et al., 2010).
In Nicotiana tabacum, the expression of the Ntmybl gene is induced during the response to Tobacco Mosaic Virus (TMV) and Pseudomonas syringae pv. syringae avirulent bacteria. The Ntmyb1 protein binds to the promoter of the defense-related gene PR-1a suggesting a role in the regulation of immune responses (Yang and Klessig, 1996). In an independent study, Ntmyb1 was retrieved together with three other R2R3 MYBs as factors binding to the promoter of defense-related genes (Sugimoto et al., 2000). Transgenic N. tabacum plants overexpressing the rubber tree HbMyb1 MYB gene exhibited suppressed HR resulting in enhanced resistance to the necrotrophic fungus Botrytis cinerea (Peng et al., 2011). Conversely, overexpression of the wheat TaPIMP1 MYB gene caused stronger HR and enhanced resistance to the biotrophic bacterial pathogen Ralstonia solanacearum in tobacco and to the hemibiotrophic fungal pathogen Bipolaris sorokiniana in wheat (Liu et al., 2011; Zhang et al., 2012). In rice, the OsJaMyb R2R3 MYB gene is induced during infection by the blast fungus Magnaporthe oryzae and in mutants altered in cell death programs suggesting a role in defense responses (Lee et al., 2001). The Arabidopsis thaliana genome harbors 137 R2R3 MYB genes some of which have been shown to regulate immunity to microbial pathogens. The BOTRYTIS-SUSCEPTIBLE1 BOS1/AtMYB108 gene was identified in a screen for mutants altered in their response to the $B$. cinerea. The bos 1 mutant exhibits enhanced susceptibility to B. cinerea and Alternaria brassicicola necrotrophic pathogens and reduced symptoms but unaltered resistance in response to biotrophic pathogens (Mengiste et al., 2003). Conversely, AtMYB46 negatively regulates resistance to $B$. cinerea likely via the regulation of a cell wall-bound 
peroxidase (Ramirez et al., 2011). Overexpression and silencing of AtMYB44 demonstrated that it positively regulates resistance to the virulent bacterium P. syringae pv. tomato (Pst) DC3000 but down regulates resistance to A. brassicicola via the WRKY70 TF (Shim et al., 2012; Zou et al., 2012). AtMYB96 was first reported as induced upon Cauliflower Mosaic Virus infection (Geri et al., 1999). Analysis of plants mis-expressing AtMYB96 demonstrated that this TF positively controls resistance to Pst DC3000 in a salicylic acid-dependent manner (Seo and Park, 2010). Among the closest paralogs of AtMYB96 is AtMYB30, which was the first R2R3 MYB gene to be associated with the regulation of defense response in Arabidopsis and one of the best defense-related MYBs characterized to date. Although the mechanisms by which MYB TFs control defense responses are still enigmatic, recent advances in our understanding of AtMYB30 function summarized in this review shed new light on the regulation of plant immunity by this family of TFs.

The MYB oncogene homologue AtMYB30 was first isolated in by differential screening of a cDNA library prepared from Xanthomonas campestris pv. campestris (Xcc)-inoculated Arabidopsis cells (Lacomme and Roby, 1999). Early, transient and specific activation of AtMYB30, prior to the onset of the hypersensitive cell death, was observed after treatment with different avirulent bacterial pathogens (Daniel et al., 1999). In addition, overexpression of AtMYB30 in Arabidopsis and tobacco led to acceleration and intensification of the HR, enhanced accumulation of HR molecular markers and increased resistance in response to avirulent pathogens. Conversely, the antisensemediated downregulation of AtMYB30 led to a strong decrease or suppression of the HR (Vailleau et al., 2002). These data identify AtMYB30 as a positive regulator of the signaling pathway controlling the establishment of cell death responses to pathogen attack.

During the last few years, the study of AtMYB30 regulatory mechanisms has increased our knowledge about the mode of action of this TF. These studies have uncovered a tight control of the activity of AtMYB30 through protein-protein interactions and post-translational modifications (PTMs). Here, we summarize our current knowledge of the AtMYB30 interaction and regulatory network involved in the control of plant defense responses. Additional roles of AtMYB30 during the integration of other environmental cues are also discussed.

\section{AtMYB30 REGULATES GENES OF THE VLCFA PATHWAY}

A transcriptomic analysis revealed that AtMYB30 putative target genes are involved in the lipid biosynthesis pathway that leads to the production of very long chain fatty acids (VLCFAs) (Raffaele et al., 2008). In good agreement, ectopic expression of AtMYB30 activates genes encoding subunits of the acyl-coA elongase complex and alters the VLCFA content of Arabidopsis leaves. Furthermore, defense-related phenotypes of AtMYB30 transgenic plants are dependent on the VLCFA biosynthesis pathway, supporting the view that AtMYB30 modulates cell death-related lipid signaling by enhancing the synthesis of VLCFAs or VLCFA derivatives (Raffaele et al., 2008) (Figure 1A). Downstream products of the VLCFA pathway include sphingolipids, wax and cutin.
Wax synthesis was altered by AtMYB30 over-expression but not by $A t M Y B 30$ silencing, suggesting that sphingolipids could be cell death signals regulated by AtMYB30, and that activators of the wax synthesis pathway could compensate for the lack of AtMYB30 in silenced plants. Interestingly, Seo et al. reported that AtMYB96 activates genes of the wax biosynthesis pathway during drought stress (Seo et al., 2011). AtMYB30 and AtMYB96 belong to the sub-group S1 of Arabidopsis R2R3 MYB family (Figure 2A) (Dubos et al., 2010). Their N-terminal domain is predicted to mediate DNA-binding through a six alpha-helix domain typical of R2R3 MYBs (Figure 2B). AtMYB30 and AtMYB96 share extensive similarity in their N-terminal domain (Figure 2C), as expected considering the overlap in their respective lists of target genes. Besides short conserved motifs, the C-termini of sub-group S1 of MYB TFs are highly divergent. In AtMYB30, this C-terminal region harbors numerous putative regulatory sites, including phosphorylation, SUMOylation and ubiquitination sites (Figure 2C). As discussed below, modifications of this kind are critical for the regulation of AtMYB30 activity. It is therefore tempting to speculate that the differential activation of the N-termini of MYB TFs of the sub-group S1 may integrate signals arising from multiple stresses to regulate a partially common set of genes. Whether and how the interplay between AtMYB30 and AtMYB96 fine-tunes the activation of VLCFAmediated responses remains to be investigated. Whether other MYBs of sub-group S1 are able to activate the VLCFA pathway is also unknown. Shared and specific functions of related MYB TFs may explain how expansion and diversification in this family contributed to the emergence of an integrated stress-response machinery in plants.

\section{MANIPULATION OF AtMYB3O ACTIVITY BY BACTERIA}

XopD from strain B100 of Xanthomonas campestris pv. campestris $(X c c B 100)$ is a modular type III effector protein of 801 amino acids that presents a modular structure and contains different domains with varied biochemical activities (Canonne et al., 2012). XopD $\mathrm{X}_{\mathrm{XcB} B 100}$ is targeted to plant cell nuclei (Canonne et al., 2011; Kim et al., 2011) and may interact with chromatin and/or transcriptional units, leading to modulation of host transcription by affecting chromatin remodeling and/or TF activity (Kay and Bonas, 2009).

In agreement with the idea that plant TFs and/or regulators might be direct targets of XopD, XopD $\mathrm{XccB}_{100}$ was shown to target AtMYB30. XopD $D_{X c c B 100}$ expression leads to accumulation of AtMYB30 in $\mathrm{XopD}_{\mathrm{XccB} 100}$-containing nuclear foci but the physical interaction between $\mathrm{XopD}_{X c c B 100}$ and AtMYB30 is independent of AtMYB30 relocalization to nuclear foci, as both proteins are also able to interact in the nucleoplasm (Canonne et al., 2011). Xop $D_{X c c B 100}$ targeting of AtMYB30 leads to reduced activation of AtMYB30 VLCFA-related target genes and, therefore, to suppression of plant defense responses during XccB100 infection (Canonne et al., 2011) (Figure 1A). A helix-loop-helix (HLH) domain in $\mathrm{XopD}_{\mathrm{XccB100}}$ is necessary and sufficient to mediate the interaction with AtMYB30 and repression of AtMYB30 transcriptional activation and plant resistance responses. Consistently, XopD from the 8004 strain of $\mathrm{Xcc}\left(\mathrm{XopD}_{\mathrm{Xcc8004}}\right)$, that does not present the HLH domain and localizes homogenously within 


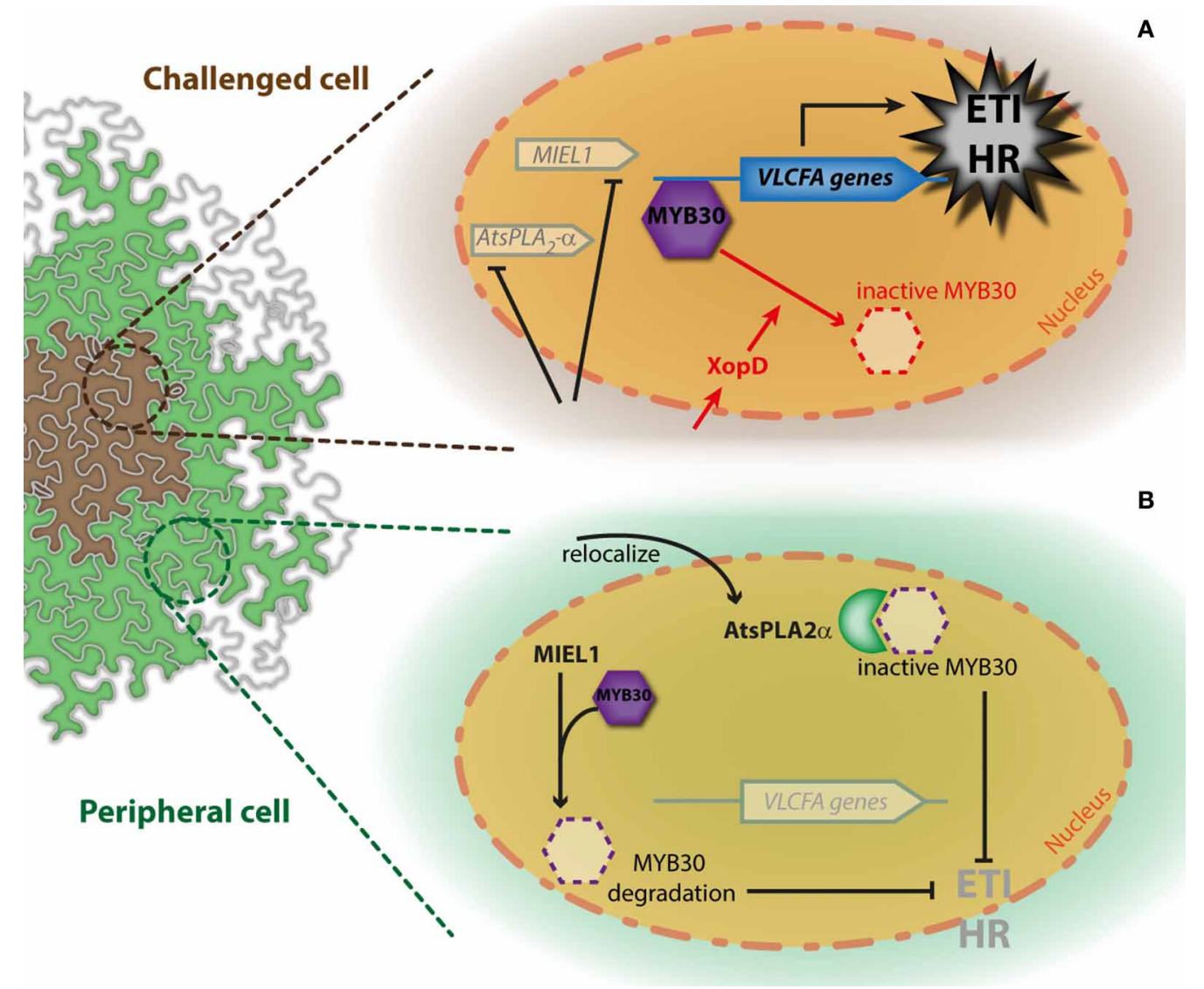

FIGURE 1 | Simplified model for the simultaneous regulation of AtMYB30-mediated $\mathrm{HR}$ cell death through interaction with AtsPLA $\mathbf{A}_{2}-\alpha$ and MIEL1. The action of with AtSPLA ${ }_{2}-\alpha$ and MIEL1 on AtMYB30-mediated
HR development is presented in cells challenged with bacterial inoculation (A) and peripheral cells (B). Activity of the bacterial XopD effector is shown in red. See the text for details. plant cell nuclei, is not able to interact with AtMYB30 and has no effect on AtMYB30 transcriptional activation. Considering the modular structure of XopD, it is likely that this type III effector mediates multiple molecular (protein-DNA and proteinprotein) associations and that, depending on the Xanthomonas strain/host plant interaction, XopD is able to target different host components to subvert plant defense. For example, XopD from Xanthomonas euvesicatoria ( $X c v$ ) desumoylates the SIERF4 TF to suppress ethylene responses and promote pathogen growth in tomato (Kim et al., 2013).

\section{REGULATION OF ATMYB3O ACTIVITY THROUGH PROTEIN-PROTEIN INTERACTIONS AND POST-TRANSLATIONAL MODIFICATIONS}

Plant resistance to disease involves costly defense responses, closely connected to plant physiological and developmental processes. A typical example is the HR, which includes the development of a form of programmed cell death and needs to be tightly regulated to be not only efficient but also beneficial to the plant. As a result, mutants with constitutively active defense responses often present stunted growth and low fertility (Lorrain et al., 2003). Negative regulatory mechanisms of defense responses are used by the plant to attenuate the activation of defense-related functions and allow a balanced allocation of resources upon pathogen challenge (Journot-Catalino et al., 2006; Mukhtar et al., 2008). AtMYB30 being a positive regulator of plant defense and associated cell death responses, several mechanisms of negative regulation of its activity have been described.

The secretory phospholipase $\mathrm{PLA}_{2}$ protein AtsPLA $-\alpha$ controls auxin transport protein trafficking to the plasma membrane (Lee et al., 2010). AtsPLA 2 - $\alpha$ localizes to Golgi-associated vesicles and is later secreted to the extracellular space (Froidure et al., 2010; Lee et al., 2010). Translocation of AtsPLA $2-\alpha$ to the apoplast is enhanced after plant inoculation with avirulent bacteria, suggesting that Ats $\mathrm{PLA}_{2}-\alpha$ may participate to the plant defense response in the apoplast (Jung et al., 2012). Interestingly, intracellular AtsPLA $2-\alpha$ has also been involved in the non-enzymatic control of plant defense. Indeed, AtsPLA $2-\alpha$ was identified as interacting with AtMYB30 in yeast (Froidure et al., 2010). In the presence of AtMYB30, AtsPLA 2 - $\alpha$ was partially relocalized to the plant cell nucleus where these two proteins interact, leading to repression of the AtMYB30-mediated transcriptional activity. As a result, Arabidopsis HR and defense responses are suppressed, supporting the view that AtMYB30 transcriptional activity is 
A
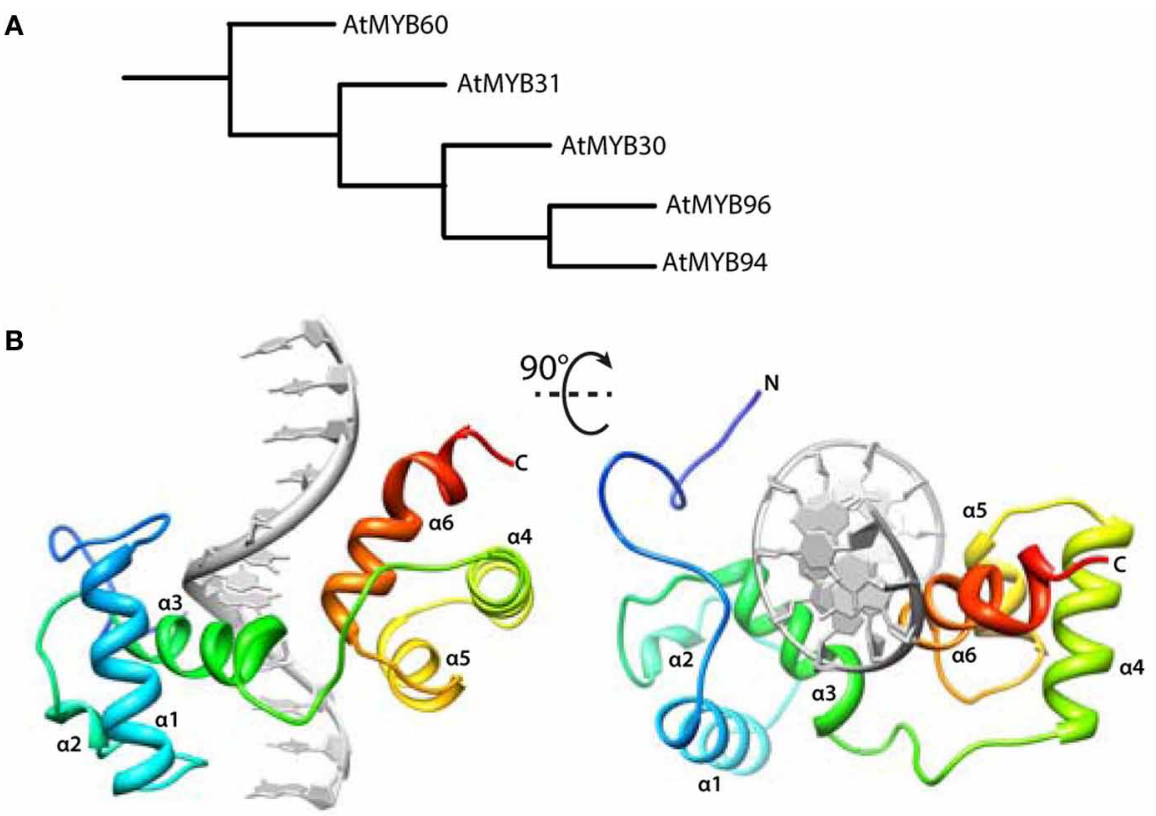

C

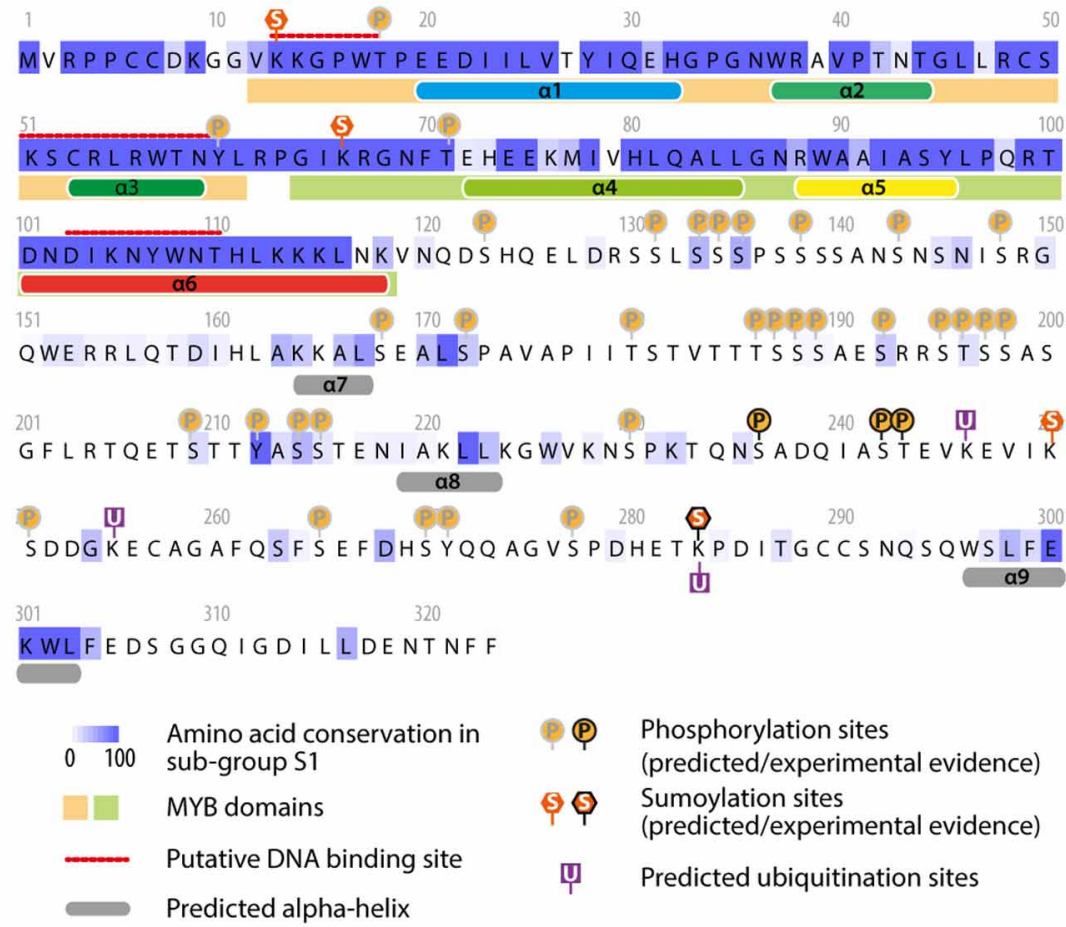

FIGURE 2 | AtMYB30 sequence analysis: relationship with other MYBs, protein motifs and predicted structure. (A) Relationship between MYB TFs of the subgroup S1 (from Dubos et al., 2010). (B) Predicted structure of AtMYB30 DNA binding domain bound to DNA (gray). The model was predicted using the I-TASSER server and rendered with UCSF Chimera. (C) Sequence analysis of AtMYB30

protein. The conservation between members of subgroup S1 was inferred from a MUSCLE alignment and colored using JALVIEW. Alpha helices and DNA binding sites were predicted using the I-TASSER server. MYB domains were identified using INTERPROSCAN. Phosphorylation, sumoylation and ubiquitation sites were predicted using PhosphAt, Sumoplot and Ubpred respectively.

required to mount an efficient defense response during bacterial infection (Raffaele et al., 2008; Froidure et al., 2010). Notably, AtsPLA $2-\alpha$ nuclear targeting, interaction with AtMYB30, repression of AtMYB30 transcriptional activity and HR development appeared to be independent of AtsPLA $2-\alpha$ enzymatic activity (Froidure et al., 2010). Therefore, AtsPLA $2-\alpha$ was proposed to control AtMYB30-mediated response through interaction with AtMYB30, preventing the activation of its targets, rather than 
through a lipid signal produced by AtsPLA $\mathrm{PL}_{2}-\alpha$. Together, these data highlight the importance of dynamic nucleocytoplasmic protein trafficking for the regulation of the transcriptional activation related to defense (Rivas, 2012). Interestingly, AtMYB30 expression is induced $4 \mathrm{~h}$ post-inoculation (hpi) in challenged cells but not in peripheral cells, whereas Ats $P L A_{2}-\alpha$ expression peaks 6 hpi in peripheral but not in challenged cells (Froidure et al., 2010). This suggests that AtsPLA $\mathrm{PL}_{2}-\alpha$ may contribute to restrict the development of the HR to the inoculated zone, thereby preventing spreading of cell death throughout the leaf (Froidure et al., 2010) (Figure 1B).

An additional regulatory mechanism of AtMYB30 action was uncovered by the identification of the Arabidopsis RING-

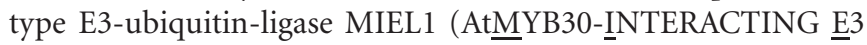
LIGASE1) as an AtMYB30 interactor in yeast (Marino et al., 2013). MIEL1 is able to ubiquitinate AtMYB30 in vitro. In Arabidopsis, MIEL1 leads to AtMYB30 proteasomal degradation, downregulation of its transcriptional activity and suppression of plant defense responses (Marino et al., 2013). Indeed, Arabidopsis miel1 mutant plants displayed enhanced $\mathrm{HR}$ and resistance after inoculation with avirulent bacteria. These phenotypes are AtMYB30-dependent and correlate with down-regulation of AtMYB30 target genes related to VLCFA metabolism (Marino et al., 2013). MIEL1 expression is rapidly repressed in challenged cells, indicating that MIEL1 may negatively regulate plant HR and defense activation through degradation of the MYB30 protein in the absence of the pathogen (Marino et al., 2013; Figure 2B). Repression of MIEL1 in challenged cells may release AtMYB30 negative regulation, increasing the intensity of the HR and limiting pathogen growth (Marino et al., 2013; Figure 2A). In addition, MIEL1-mediated degradation of AtMYB30 could contribute to the spatial restriction of the HR to inoculated cells since MIEL1 expression remains constant in peripheral cells (Marino et al., 2013; Figure 2B). Work by Marino and co-workers shows the important role played by ubiquitination during the transcriptional control of the HR (Marino et al., 2012) and underlines the sophisticated fine-tuning of plant responses to pathogen attack.

PTM of AtMYB30 by SUMOylation has also been reported. AtMYB30 SUMOylation was first demonstrated after reconstitution of the SUMOylation cascade in E. coli, the lysine residue K283 being the major SUMOylation site (Okada et al., 2009; Figure 2C). SUMOylation of AtMYB30 K283 by the Arabidopsis SUMO E3 ligase SIZ1 was later confirmed in Arabidopsis protoplasts and demonstrated to be required for AtMYB30 function during abscisic acid (ABA) signaling (Zheng et al., 2012) (see below). However, whether and how SUMOylation of AtMYB30 affects AtMYB30-mediated defense responses remains to be determined.

Finally, the AtMYB30 C-terminal region is particularly rich in potential phosphorylation sites for several protein kinases (Figure 2C). The contribution of these phosphorylation sites to the plant defense response is still unknown but it is tempting to speculate that different combinations of PTMs on AtMYB30 may act as a molecular barcode, which would be important for the regulation of TFs controlling multiple processes (Benayoun and Veitia, 2009). Along these lines, the animal TFs p53 and c-Myc represent excellent paradigms that illustrate the sophistication of transcription regulation with different PTMs providing efficient regulation of TF stability, subcellular localization and activity (Meek and Anderson, 2009; Hammond-Martel et al., 2012; Luscher and Vervoorts, 2012).

\section{AtMYB30, A REGULATOR OF MULTIPLE SIGNALS BEYOND THE RESPONSE TO MICROBES}

In addition to its role as a positive regulator of defense responses, AtMYB30 is recruited for the regulation of other signaling processes. The phytohormone ABA plays an essential role during development and in response to abiotic and biotic stress. AtMYB30 SUMOylation by SIZ1 leads to AtMYB30 protein stabilization and affects AtMYB30-mediated transcriptional activation of several ABA-responsive genes (Zheng et al., 2012), underlining the importance of AtMYB30 SUMOylation during the regulation of ABA signaling. As a result, an atmyb30 mutant is hypersensitive to $A B A$ whereas AtMYB30-overexpressing plants are insensitive to ABA (Zheng et al., 2012). Conversely, AtMYB96 overexpressing plants were found to be hypersensitive to ABA, but an atmyb96 knockout mutant was still responsive to ABA, possibly due to functional redundancy within the MYB family (Seo et al., 2009). AtMYB96 expression is induced by ABA and drought and the activation of some ABA-inducible genes is AtMYB96-dependent. Similar to AtMYB30, enhanced disease resistance conferred by AtMYB96 involves salicylic acid synthesis, suggesting that these two MYB TFs regulate cross-talks between hormone signaling pathways and contribute to the integration of signals originating from various stresses (Raffaele et al., 2006; Seo and Park, 2010).

An additional example of the diversity of AtMYB30 functions is the regulation of brassinosteroid (BR) signaling. BRs play important roles in several plant growth and developmental processes as well as during stress/disease resistance. BRs signal through the BES1 (bri1-ethylmethane sulphonate suppressor1)/BZR1 (brassinazole-resistant1) family of TFs. BR treatment induces AtMYB30 gene expression in Arabidopsis seedlings and in bes1-D plants, that overexpress BES1, AtMYB30 expression is upregulated, indicating that AtMYB30 may function in the BR signaling pathway (Li et al., 2009). Indeed, chromatin immunoprecipitation (ChIP) experiments showed that BES1 activates AtMYB30 expression by directly binding to the AtMYB30 promoter (Li et al., 2009). In agreement with this finding, atmyb30 knockout mutant plants exhibit reduced BR-related gene expression and phenotypes, indicating that AtMYB30 promotes the expression of a subset of BR target genes (Li et al., 2009). Moreover, the promoters of AtMYB30 and BES1 common target genes harbor boxes bound by each TF. Finally, AtMYB30 and BES1 interact with each other. Together, this data shows that AtMYB30 functions to amplify BR signaling through cooperation with BES1 to promote BR target gene expression.

\section{CONCLUSIONS AND PERSPECTIVES}

Cellular responses to environmental or physiological cues rely on transduction pathways that must discriminate between different signals and ensure a combinatorial regulation. Thus, combinations of different PTMs and protein-protein interactions provide different layers of information that may allow the 
integration of several transduction pathways and warrant highly specific cellular outputs. Accumulating evidence shows that the Arabidopsis MYB regulator AtMYB30 is a multi-regulated protein that is involved in the integration of various environmental stimuli, including attack by microbes, abiotic stress and hormone signaling, likely through the activation of shared and specific sets of target genes. How simultaneous and diverse stress signals are integrated into a unified cellular response is a major unknown in cell signaling. The acceleration of large data set acquisition

\section{REFERENCES}

Benayoun, B. A., and Veitia, R. A. (2009). A post-translational modification code for transcription factors: sorting through a sea of signals. Trends Cell Biol. 19, 189-197.

Canonne, J., Marino, D., Jauneau, A., Pouzet, C., Briere, C., Roby, D., et al. (2011). The Xanthomonas type III effector XopD targets the Arabidopsis transcription factor AtMYB30 to suppress plant defence. Plant Cell 23, 3498-3511.

Canonne, J., Pichereaux, C., Marino, D., Roby, D., Rossignol, M., and Rivas, S. (2012). Identification of the protein sequence of the type III effector XopD from the B100 strain of Xanthomonas campestris pv. campestris. Plant Signal. Behav. 7, 184-187.

Coll, N. S., Epple, P., and Dangl, J. L. (2011). Programmed cell death in the plant immune system. Cell Death Differ. 18, 1247-1256.

Daniel, X., Lacomme, C., Morel, J.-B., and Roby, D. (1999). A novel $m y b$ oncogene homolog in Arabidopsis thaliana related to the hypersensitive cell death. Plant J. 20, 57-66.

Dias, A. P., Braun, E. L., McMullen, M. D., and Grotewold, E. (2003). Recently duplicated maize R2R3 Myb genes provide evidence for distinct mechanisms of evolutionary divergence after duplication. Plant Physiol. 131, 610-620.

Dubos, C., Stracke, R., Grotewold, E., Weisshaar, B., Martin, C., and Lepiniec, L. (2010). MYB transcription factors in Arabidopsis. Trends Plant Sci. 15, 573-581.

Feller, A., Machemer, K., Braun, E. L., and Grotewold, E. (2011). Evolutionary and comparative analysis of MYB and bHLH plant transcription factors. Plant J. 66, 94-116.

Froidure, S., Canonne, J., Daniel, X., Jauneau, A., Briere, C., Roby, D., et al. (2010). AtsPLA2-alpha nuclear relocalization by the Arabidopsis transcription factor AtMYB30 leads to repression of the plant defense response. Proc. Natl. Acad. Sci. U.S.A. 107, 15281-15286.
Geri, C., Cecchini, E., Giannakou, M. E., Covey, S. N., and Milner, J. J. (1999). Altered patterns of gene expression in Arabidopsis elicited by cauliflower mosaic virus (CaMV) infection and by a CaMV gene VI transgene. Mol. Plant Microbe Interact. 12, 377-384.

Hammond-Martel, I., Yu, H., and Affar el, B. (2012). Roles of ubiquitin signaling in transcription regulation. Cell. Signal. 24, 410-421.

Jones, J. D., and Dangl, J. L. (2006). The plant immune system. Nature 444, 323-329.

Journot-Catalino, N., Somssich, I. E., Roby, D., and Kroj, T. (2006). The transcription factors WRKY11 and WRKY17 act as negative regulators of basal resistance in Arabidopsis thaliana. Plant Cell 18, 3289-3302.

Jung, J., Kumar, K., Lee, H. Y., Park, Y. I., Cho, H. T., and Ryu, S. B. (2012). Translocation of phospholipase A2alpha to apoplasts is modulated by developmental stages and bacterial infection in Arabidopsis. Front. Plant Sci. 3:126. doi: 10.3389/fpls.2012.00126

Kay, S., and Bonas, U. (2009). How Xanthomonas type III effectors manipulate the host plant. Curr. Opin. Microbiol. 12, 37-43.

Kim, J. G., Stork, W., and Mudgett, M. B. (2013). Xanthomonas type III effector XopD desumoylates tomato transcription factor SIERF4 to suppress ethylene responses and promote pathogen growth. Cell Host Microbe 13, 143-154.

Kim, J. G., Taylore, K. W., and Mudgett, M. B. (2011). Comparative analysis of the XopD type III secretion (T3S) effector family in plant pathogenic bacteria. Mol. Plant Pathol. 12, 715-730.

Lacomme, C., and Roby, D. (1999). Identification of new early markers of the hypersensitive response in Arabidopsis thaliana. FEBS Lett. 459, 149-153.

Lee, M. W., Qi, M., and Yang, Y. (2001). A novel jasmonic acidinducible rice $M Y B$ gene associates with fungal infection and host cell

and the development of systems biology approaches promise to offer new insights into the functioning of such complex regulatory networks. The wealth of knowledge gained in recent years on Arabidopsis R2R3 MYB TFs provides an excellent framework toward this end.

\section{ACKNOWLEDGMENTS}

Our work is performed at the LIPM that is part of the Laboratoire d'Excellence (LABEX) entitled TULIP (ANR-10-LABX-41).

death. Mol. Plant Microbe Interact. 14, 527-535.

Lee, O., Kim, S., Kim, H., Hong, J., Ryu, S., Lee, S., et al. (2010). Phospholipase A2 is required for PIN-FORMED protein trafficking to the plasma membrane in the Arabidopsis root. Plant Cell 22, 1812-1825.

Li, L., Yu, X., Thompson, A., Guo, M., Yoshida, S., Asami, T., et al. (2009). Arabidopsis MYB30 is a direct target of BES1 and cooperates with BES1 to regulate brassinosteroidinduced gene expression. Plant J. 58, 275-286.

Liu, H., Zhou, X., Dong, N., Liu, X., Zhang, H., and Zhang, Z. (2011). Expression of a wheat MYB gene in transgenic tobacco enhances resistance to Ralstonia solanacearum, and to drought and salt stresses. Funct. Integr. Genomics 11, 431-443.

Lorrain, S., Vailleau, F., Balagué, C. and Roby, D. (2003). Lesion mimic mutants: keys for deciphering cell death and defense pathways in plants? Trends Plant Sci. 8, 263-271.

Luscher, B., and Vervoorts, J. (2012). Regulation of gene transcription by the oncoprotein MYC. Gene 494, 145-160.

Marino, D., Froidure, S., Canonne, J., Ben Khaled, S., Khafif, M., Pouzet, C., et al. (2013). Arabidopsis ubiquitin ligase MIEL1 mediates degradation of the transcription factor MYB30 weakening plant defence. Nat. Commun. 4, 1476.

Marino, D., Peeters, N., and Rivas, S. (2012). Ubiquitination during plant immune signaling. Plant Physiol. 160, 15-27.

Meek, D. W., and Anderson, C. W. (2009). Posttranslational modification of p53: cooperative integrators of function. Cold Spring Harb. Perspect. Biol. 1:a000950. doi: 10.1101/cshperspect.a000950

Mengiste, T., Chen, X., Salmeron, J., and Dietrich, R. (2003). The BOTRYTIS SUSCEPTIBLE1 gene encodes an R2R3MYB transcription factor protein that is required for biotic and abiotic stress responses in Arabidopsis. Plant Cell 15, 2551-2565.

Moore, J. W., Loake, G. J., and Spoel, S. H. (2011). Transcription dynamics in plant immunity. Plant Cell 23, 2809-2820.

Mukhtar, M. S., Deslandes, L., Auriac, M. C., Marco, Y., and Somssich, I. E. (2008). The Arabidopsis transcription factor WRKY27 influences wilt disease symptom development caused by Ralstonia solanacearum. Plant J. 56, 935-947.

Mur, L. A., Kenton, P., Lloyd, A. J., Ougham, H., and Prats, E. (2008). The hypersensitive response; the centenary is upon us but how much do we know? J. Exp. Bot. 59, 501-520.

Okada, S., Nagabuchi, M., Takamura, Y., Nakagawa, T., Shinmyozu, K., Nakayama, J., et al. (2009). Reconstitution of Arabidopsis thaliana SUMO pathways in E. coli: functional evaluation of SUMO machinery proteins and mapping of SUMOylation sites by mass spectrometry. Plant Cell Physiol. 50, 1049-1061.

Peng, S. Q., Wu, K. X., Huang, G. X., and Chen, S. C. (2011). HbMyb1, a Myb transcription factor from Hevea brasiliensis, suppresses stress induced cell death in transgenic tobacco. Plant Physiol. Biochem. 49, 1429-1435.

Raffaele, S., Rivas, S., and Roby, D. (2006). An essential role for salicylic acid in AtMYB30-mediated control of the hypersensitive cell death program in Arabidopsis. FEBS Lett. 580, 3498-3504.

Raffaele, S., Vailleau, F., Leger, A., Joubes, J., Miersch, O., Huard, C., et al. (2008). A MYB transcription factor regulates Very-Long-Chain Fatty Acid biosynthesis for activation of the hypersensitive cell death response in Arabidopsis. Plant Cell 20, 752-767.

Ramirez, V., Agorio, A., Coego, A., Garcia-Andrade, J., Hernandez, M. J., Balaguer, B., et al. (2011). MYB46 modulates disease susceptibility to Botrytis cinerea in Arabidopsis. Plant Physiol. 155, 1920-1935. 
Rivas, S. (2012). Nuclear dynamics during plant innate immunity. Plant Physiol. 158, 87-94.

Seo, P. J., Lee, S. B., Suh, M. C., Park, M. J., Go, Y. S., and Park, C. M. (2011). The MYB96 transcription factor regulates cuticular wax biosynthesis under drought conditions in Arabidopsis. Plant Cell 23, 1138-1152.

Seo, P. J., and Park, C. M. (2010). MYB96-mediated abscisic acid signals induce pathogen resistance response by promoting salicylic acid biosynthesis in Arabidopsis. New Phytol. 186, 471-483.

Seo, P. J., Xiang, F., Qiao, M., Park, J. Y., Lee, Y. N., Kim, S. G., et al. (2009). The MYB96 transcription factor mediates abscisic acid signaling during drought stress response in Arabidopsis. Plant Physiol. 151, 275-289.

Shim, J. S., Jung, C., Lee, S., Min, K., Lee, Y. W., Choi, Y., et al. (2012). AtMYB44 regulates WRKY70 expression and modulates antagonistic interaction between salicylic acid and jasmonic acid signaling. Plant J. 73, 483-495.

Shiu, S. H., Shih, M. C., and Li, W. H. (2005). Transcription factor families have much higher expansion rates in plants than in animals. Plant Physiol. 139, 18-26.

Stracke, R., Werber, M., and Weisshaar, B. (2001). The R2R3-MYB gene family in Arabidopsis thaliana. Curr. Opin. Plant Biol. 4, 447-456.

Sugimoto, K., Takeda, S., and Hirochika, H. (2000). MYBrelated transcription factor NtMYB2 induced by wounding and elicitors is a regulator of the tobacco retrotransposon Ttol and defense-related genes. Plant Cell 12, 2511-2527.

Vailleau, F., Daniel, X., Tronchet, M., Montillet, J. L., Triantaphylides, C., and Roby, D. (2002). A R2R3-MYB gene, AtMYB30, acts as a positive regulator of the hypersensitive cell death program in plants in response to pathogen attack. Proc. Natl. Acad. Sci. U.S.A. 99, 10179-10184.

Yang, Y., and Klessig, D. F. (1996). Isolation and characterization of a tobacco mosaic virus-inducible $m y b$ oncogen homolog from tobacco. Proc. Natl. Acad. Sci. U.S.A. 93, 14972-14977.

Zhang, Z., Liu, X., Wang, X., Zhou, M., Zhou, X., Ye, X., et al. (2012). An R2R3 MYB transcription factor in wheat, TaPIMP1, mediates host resistance to Bipolaris sorokiniana and drought stresses through regulation of defense- and stress-related genes. New Phytol. 196, 1155-1170.

Zheng, Y., Schumaker, K. S., and Guo, Y. (2012). Sumoylation of transcription factor MYB30 by the small ubiquitin-like modifier E3 ligase SIZ1 mediates abscisic acid response in Arabidopsis thaliana. Proc. Natl. Acad. Sci. U.S.A. 109, 12822-12827.

Zou, B., Jia, Z., Tian, S., Wang, X. Gou, Z., Lü, B., et al. (2012). AtMYB44 positively modulates disease resistance to Pseudomonas syringae through the salicylic acid signalling pathway in Arabidopsis. Funct. Plant Biol. 40, 304-313.

Conflict of Interest Statement: The authors declare that the research was conducted in the absence of any commercial or financial relationships that could be construed as a potential conflict of interest.

Received: 04 March 2013; paper pending published: 21 March 2013; accepted: 28 March 2013; published online: 11 April 2013.

Citation: Raffaele S and Rivas S (2013) Regulate and be regulated: integration of defense and other signals by the AtMYB30 transcription factor. Front. Plant Sci. 4:98. doi: 10.3389/fpls. 2013.00098

This article was submitted to Frontiers in Plant-Microbe Interaction, a specialty of Frontiers in Plant Science.

Copyright (c) 2013 Raffaele and Rivas. This is an open-access article distributed under the terms of the Creative Commons Attribution License, which permits use, distribution and reproduction in other forums, provided the original authors and source are credited and subject to any copyright notices concerning any third-party graphics etc. 